\title{
Planeamiento de la Expansión de la Red de Transmisión en Sistemas Eléctricos de Potencia Considerando Reconfiguraciones de Red
}

\author{
Diego Tejada-Arango ${ }^{(1)}$, Jesús M. López-Lezama ${ }^{(2)}$ y Marcos J. Rider ${ }^{(3)}$ \\ (1) XM S.A. E.S.P., Calle 12 Sur No. 18 - 168 Bloque 2, Medellín-Colombia \\ (e-mail: dtejada@xm.com.co) \\ (2) Universidad de Antioquia, Grupo GIMEL, Departamento de Ingeniería Eléctrica, Facultad de Ingeniería, \\ Calle 70 No.52-21, Medellín, Colombia (e-mail: lezama@udea.edu.co) \\ (3) Universidade Estadual Paulista (UNESP), Faculdade de Engenharia, Av. Brasil 56 Centro, llha Solteira- \\ Brasil (e-mail: mjrider@dee.feis.unesp.br)
}

Recibido Jun. 19, 2013; Aceptado Jul. 31, 2013; Versión final recibida Ago. 8, 2013

\begin{abstract}
Resumen
En este artículo se describen las ecuaciones necesarias para que las reconfiguraciones de una red de transmisión existente puedan ser consideradas en el modelo lineal disyuntivo utilizado para resolver el problema de planeación de la red. En el método tradicional usado para resolver este problema se emplean como candidatos de expansión líneas de transmisión y transformadores de dos devanados. Sin embargo, la reconfiguración de la red existente no ha sido considerada como una solución válida en los modelos clásicos. Para mostrar que la consideración de las reconfiguraciones de red pueden reducir los costos de inversión del problema de planeamiento de la red de transmisión se utiliza el sistema de prueba Garver y un sistema colombiano. El trabajo muestra que contar con un modelo de optimización que incluya candidatos convencionales y reconfiguraciones permite dar mejores señales de expansión en las redes de transmisión de sistemas eléctricos de potencia.
\end{abstract}

Palabras clave: red de transmisión, sistemas eléctricos de potencia, reconfiguraciones de red, programación lineal

\section{Transmission Expansion Planning in Electrical Power Systems Considering Network Reconfiguration}

\begin{abstract}
In this paper the necessary equations for considering existing network reconfiguration in the disjunctive linear model used to solve the problem transmission expansion planning. In the traditional methods used for solving this problem new transmission lines and two-winding transformers are commonly used as expansion candidates. However, reconfiguration of existing network has not been considered as a valid solution in the classical models. The Garver test system and a Colombian system are used to show that considering network reconfiguration can reduce investment costs of the transmission expansion planning problem. This work shows that an optimization model that includes conventional candidates and reconfiguration produces better expansion signals in the transmission lines of electric power systems.
\end{abstract}

Keywords: transmission network expansion planning, mixed Integer linear programming, combinatorial optimization, network reconfiguration. 


\section{INTRODUCCIÓN}

El problema de Planeamiento de la Expansión de la Red de Transmisión (PERT) es un problema clásico de los sistemas de energía eléctrica. El objetivo del PERT consiste en encontrar el plan óptimo de expansión de líneas y/o transformadores que deben ser instalados en la red para permitir la operación viable en un horizonte de tiempo predefinido, a un mínimo costo. El plan óptimo de expansión debe definir dónde, cuántos y cuándo los nuevos elementos de red (líneas y transformadores) deben ser instalados. Los datos necesarios para su solución son: topología actual, circuitos candidatos, generación, demanda para el horizonte de tiempo predefinido y restricciones de inversión, entre otros.

Un modelo matemático ideal del PERT debería utilizar las ecuaciones de flujo de carga de corriente alterna (CA) (Rider et al., 2007). Sin embargo, para resolver el planeamiento de la red de transmisión a largo plazo, es frecuente el uso de modelos matemáticos relajados. Dichos modelos suelen considerar apenas la parte activa de la operación de un sistema de energía eléctrica (potencia activa y ángulo de fase), es decir, utilizan una versión relajada del modelo CA. Los modelos principales, reportados en la literatura especializada y recopilados en (Romero et al., 2002; Latorre et al., 2003; Molina y Rudnick, 2010) son: i) El modelo de transportes (Garver, 1970); ii) El modelo híbrido lineal (Villasana et al., 1985); iii) El modelo de corriente continua (modelo CC); y iv) El modelo lineal disyuntivo (Bahiense et al., 2001).

A partir de estos modelos se han realizado nuevas propuestas que consideran planeamiento dinámico multi etapa (Escobar et al., 2001; Chen et al., 2005; Romero et al., 2003; Vinasco et al., 2011), incertidumbre en la demanda (Silvia et al., 2006; Peng et al., 2008) o restricciones de seguridad (criterio N-1) (Silvia et al., 2005; Sharifnia y Aashtiani, 1985; Kazerooni y Mulate, 2010; Zhang et al., 2012; Oliveira et al., 2007). Para la solución de estos modelos matemáticos se han planteado en la literatura diferentes técnicas de optimización tales como heurísticas, meta heurísticas y programación matemática. Mediante técnicas de optimización heurística se tiene por ejemplo el trabajo reportado en (Villasana et al., 1985).

En la optimización mediante meta heurísticas se tienen múltiples estrategias de solución para el PERT, algunos de los más usados han sido algoritmos genéticos (Gallego et al., 1998; Luiz da Silva et al., 2000; Gallego et al., 2009; Silvia et al., 2006; Escobar et al., 2001; García et al., 2012; Anaut et al., 2009) y búsqueda tabú (Areiza et al., 2001; Gallego et al., 2000; Romero et al., 2012). En cuanto a la programación matemática se han utilizado técnicas como optimización lineal (Linear Programming - LP) (Garver, 1970), optimización lineal entera mixta (Mixed Integer Linear Programming - MILP) (Bahiense et al., 2001; Vinasco et al., 2011; Pozo et al., 2013; Zhang et al., 2012) y optimización no lineal (Non Linear Programming - NLP) (Rider et al., 2007; Rider et al., 2008).

Sin embargo, aún con estos modelos y técnicas de solución, existe una brecha entre los candidatos y soluciones que se han implementado en la realidad de los sistemas de potencia y los que presentan estos modelos. La práctica ha mostrado que existen soluciones reales de menor inversión que las planteadas por los modelos clásicos al incluir en las alternativas de expansión reconfiguraciones de la red existente. Algunas aplicaciones de soluciones reales mediante reconfiguraciones de la red en sistemas de potencia, para dos casos en Colombia y uno en Canadá son: (1) Reconfiguración del circuito Guavio-Tunal 230kV en Guavio-Suria 230kV y Suria-Tunal 230kV en Colombia. (UPME, 2012); (2) Reconfiguración del circuito Guajira-Santa Marta 230kV en Guajira-Termocol 230kV y Termocol-Santa Marta 230kV en Colombia. (UPME, 2012); y (3) Proyecto de reconfiguración de línea "Windy Flats 138kV". (ALTALINK, 2013)

La principal motivación para recurrir a reconfiguraciones de la red existente, ha sido el aumento en las restricciones ambientales y la dificultad para adquirir servidumbres en los grandes centros de consumo (ej. ciudades, centros industriales) que se vienen presentando en los últimos años en el mundo, y que en ocasiones hacen que este tipo de soluciones sean la única alternativa viable de expansión de la red de transmisión. Por esta razón, a partir de la estrategia de solución al problema de planeamiento de la transmisión planteado en (Vinasco et al., 2011), en este trabajo se pretende contribuir con un modelo que permita incluir en el PERT candidatos de expansión no convencionales, como las reconfiguraciones de red. De esta forma, el nuevo modelo permite la exploración de un espacio de búsqueda más amplio, y es más coherente con soluciones que son comunes en la práctica. Es importante mencionar que en la revisión del estado del arte, no fueron identificados trabajos previos en los cuales se abordara la inclusión de reconfiguraciones en la red existente al problema de PERT. De ahí la importancia del aporte de este trabajo al estado del arte en el solución del problema de PERT.

\section{FORMULACIÓN MATEMÁTICA}

En el numeral anterior se han mencionado los diferentes modelos matemáticos para la solución del PERT. El modelo lineal disyuntivo presentado en (Bahiense et al., 2001), es un modelo equivalente lineal del 
modelo CC, por tanto es un modelo que puede ser resuelto mediante MILP conservando la primera y segunda ley de Kirchhoff. Sin embargo, para la solución con el modelo lineal disyuntivo es necesario determinar el valor del parámetro "M"; en (Vinasco et al., 2011) se establece una estrategia de solución para el modelo lineal disyuntivo asociando el parámetro "M" con el parámetro eléctrico " $2 \theta$ ", es decir, con dos veces el ángulo máximo que puede existir en una barra de un sistema eléctrico. En las ecuaciones (1) a (12) se presenta el modelo lineal disyuntivo considerando la estrategia de solución mencionada. La propuesta de inclusión de candidatos mediante reconfiguraciones se realizará sobre estas ecuaciones.

Minimizar: $v={ }_{i j \in \Omega_{l}} c_{i j} w_{i j, y}$

Sujeto a:

$$
\begin{aligned}
& f_{j i}^{0}+f_{y \in Y} f_{j i, y}-f_{i j \in \Omega_{l}} f_{i j}^{0}+f_{y \in Y} f_{i j, y}+g_{i}=d_{i} \quad \forall i \in \Omega_{b} \\
& f_{i j}^{0}=S_{b a s e} n_{i j}^{0} \frac{\theta_{i}-\theta_{j}}{x_{i j}^{p u}} \quad \forall i j \in \Omega_{l} \\
& -2 \theta 1-w_{i j, y} \leq \frac{f_{i j, y} x_{i j}^{p u}}{S_{b a s e}}-\theta_{i}-\theta_{j} \leq 2 \theta 1-w_{i j, y} \quad \forall i j \in \Omega_{l}, \forall y \in Y \\
& -n_{i j}^{0} f_{i j} \leq f_{i j}^{0} \leq n_{i j}^{0} f_{i j} \quad \forall i j \in \Omega_{l} \\
& -w_{i j, y} f_{i j} \leq f_{i j, y} \leq w_{i j, y} f_{i j} \quad \forall i j \in \Omega_{l}, \forall y \in Y \\
& 0 \leq g_{i} \leq g_{i} \quad \forall i \in \Omega_{b} \\
& -\theta \leq \theta_{i} \leq \theta \quad \forall i \in \Omega_{b} \\
& w_{i j, y} \leq n_{i j} \quad \forall i j \in \Omega_{l} \\
& w_{i j, y} \leq w_{i j, y-1} \quad \forall i j \in \Omega_{l}, \forall y \in Y / y>1 \\
& w_{i j, y} \text { binario } \quad \forall i j \in \Omega_{l}, \forall y \in Y
\end{aligned}
$$

Los elementos de este modelo (conjuntos, parámetros y variables) son los siguientes:

Conjuntos:

$\Omega_{b}$ : Conjunto de barras

$\Omega_{l}$ : Conjunto de corredores

Parámetros:

$d_{i}$ : Demanda en la barra i

$g_{i}$ : Generación máxima en la barra i 
$c_{i j}$ : Costo de construcción del corredor ij

$f_{i j}$ : Flujo de potencia máxima en el corredor ij

$n_{i j}^{0}$ : Número de elementos existentes en el corredor ij

$n_{i j}$ : Número máximo de elementos que se pueden adicionar al corredor ij

$x_{i j}^{p u}$ : Reactancia en el corredor ij

$S_{\text {base }}:$ Potencia Base

$\theta$ : Ángulo de fase máximo

\section{Variables:}

$w_{i j, y}$ : Número de líneas adicionadas en el corredor ij y circuito y

$g_{i}$ : Generación de potencia activa en la barra i

$\theta_{i}$ : Ángulo de fase en la barra $\mathrm{i}$

$f_{i j}^{0}$ : Flujo de potencia activa de los elementos existentes en el corredor ij

$f_{i j, y}$ : Flujo de potencia activa de los elementos adicionados en el corredor ij y circuito y

La función objetivo, dada por la ecuación (1), consiste en la minimización del costo de adicionar nuevas líneas. Este problema está sujeto a las restricciones (2)-(12). La restricción dada por (2) consiste en la ecuación de balance de nodos (primera ley de Kirchhoff), la restricción dada por (3) consiste en la segunda ley de Kirchhoff para las líneas existentes, la restricción (4) representa la segunda ley de Kirchhoff para las líneas candidatas. Las restricciones (5) y (6) representan el flujo máximo por las líneas existentes y candidatas, respectivamente. La restricción dada por (7) consiste en la generación máxima de cada generador del sistema. La restricción dada por (8) representa los límites máximos de los ángulos de las barras. La restricción (9) limita el número de líneas nuevas a un número máximo de elementos en el corredor ij. La restricción dada por (10) evita explorar dos veces soluciones de expansión de la red de transmisión equivalentes. La restricción (11) representa la naturaleza binaria de las variables de decisión para la inversión. Finalmente, la restricción dada por (12) indica que el ángulo de la barra de referencia debe ser cero.

\section{Modelo de reconfiguración de redes existentes en el PERT}

La reconfiguración es una opción de expansión de la red de transmisión en la cual se aprovecha parte de la red existente. En general, una línea existente es seccionada para convertirse en dos líneas nuevas las cuales están formadas por tramos de línea nuevos y tramos de la línea existente en la red inicial. En la figura 1, se ilustra esta situación.

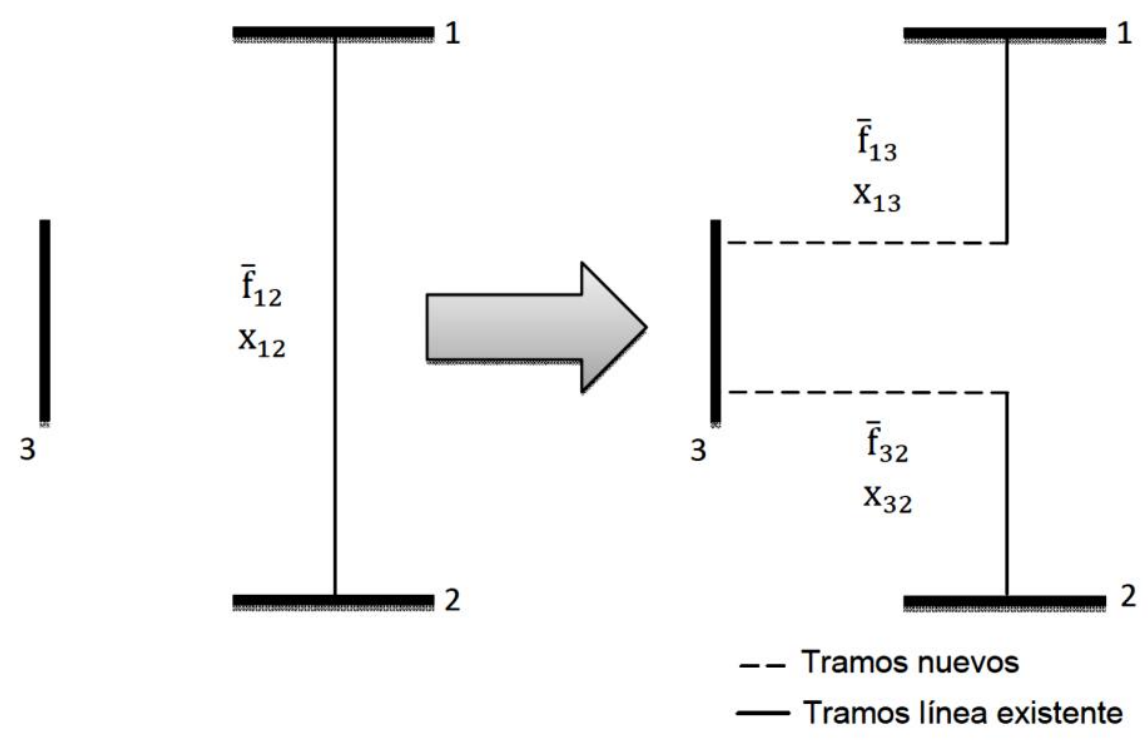

Fig. 1: Problema de la reconfiguración de una línea existente.

En la figura 1 se tiene una línea existente que une las barras 1 y 2 , la cual tiene asociada una impedancia $\left(\mathrm{x}_{12}\right)$ y capacidad máxima $\left(\mathrm{f}_{12}\right)$ dadas. Se desea conectar la barra 3 de tal forma que se aproveche gran parte de la línea existente sin conexiones en derivación o T. Una reconfiguración de la línea existente permite tener en cuenta candidatos de expansión de menor costo dentro de las alternativas de expansión de un sistema de potencia. 


\section{Modelo matemático propuesto para reconfiguración}

Un modelo matemático para considerar la reconfiguración de líneas de transmisión en la modificación al modelo lineal disyuntivo propuesta en (Vinasco et al., 2011), se desarrolla a continuación, introduciendo dos barras ficticias a y $b$ al problema en el punto donde se desea reconfigurar la línea existente tal como se presenta en la figura 2. Además, para incluir en el modelo propuesto la opción de la reconfiguración de una línea existente es necesario que se cumplan las restricciones planteadas en la tabla 1.

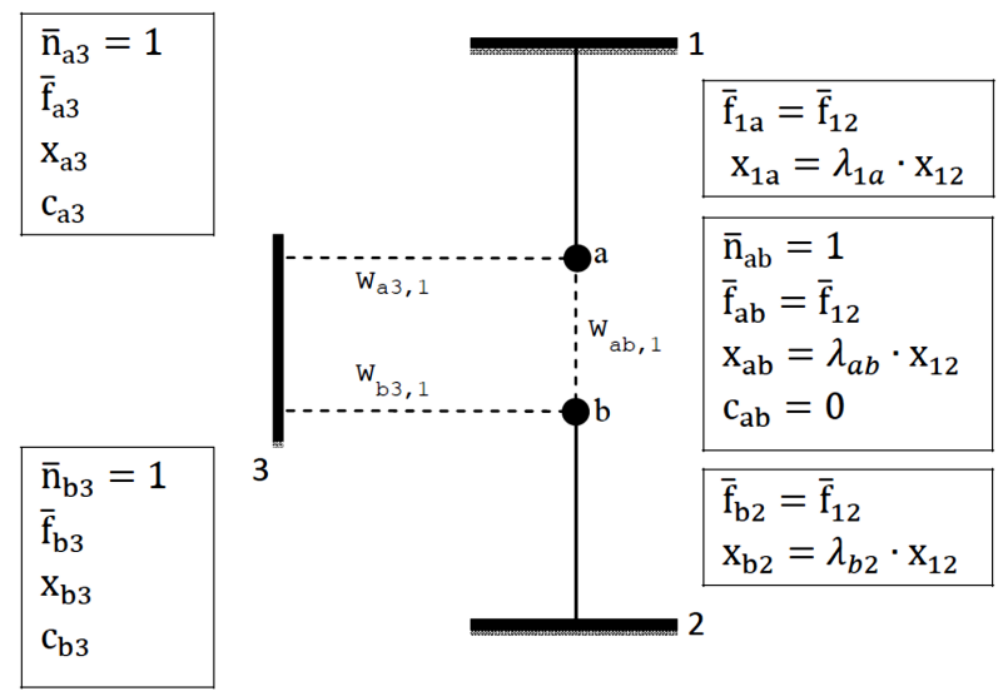

Fig. 2: Variables y parámetros para el modelo de reconfiguración.

Tabla 1. Restricciones para el modelo de reconfiguración.

\begin{tabular}{|c|l|}
\hline Restricción & \multicolumn{1}{|c|}{ Descripción } \\
\hline $\mathrm{w}_{\mathrm{a} 3,1}=\mathrm{w}_{\mathrm{b} 3,1}$ & $\begin{array}{l}\text { Garantiza que al reconfigurar estén siempre los dos tramos } \\
\text { desde la barra 3 hasta el tramo ab }\end{array}$ \\
\hline $\mathrm{w}_{\mathrm{ab}, 1}=1-\mathrm{w}_{\mathrm{a} 3,1}$ & $\begin{array}{l}\text { Restricción de complementariedad, es decir, si decide } \\
\text { reconfigurar }\left(\mathrm{w}_{\mathrm{a} 3,1}=1\right) \text { el tramo ab no debe ser } \\
\text { considerado }\left(\mathrm{w}_{\mathrm{ab}, 1}=0\right) \text { y viceversa. }\end{array}$ \\
\hline
\end{tabular}

De acuerdo a la formulación matemática presentada en la tabla 1, es posible extender el modelo lineal disyuntivo para que considere las reconfiguraciones de líneas como candidatos de expansión. Para ello es necesario definir primero las líneas de la reconfiguración en el conjunto de corredores del modelo. Además, es necesario definir un nuevo conjunto $\left(\Omega_{k}\right)$ con los nodos que permiten la reconfiguración de una línea existente a, b y e (El nodo "e" es la generalización del nodo 3 presentado en figura 2). Las ecuaciones (13) y (14) corresponden a las restricciones generalizadas de la tabla 1:

$w_{a_{k} e_{k}, 1}=w_{b_{k} e_{k}, 1}$

$\forall k \in \Omega_{k}$

$w_{a_{k} b_{k}, 1}=1-w_{a_{k} e_{k}, 1}$

$\forall k \in \Omega_{k}$

\section{Conjuntos:}

$\Omega_{\mathrm{k}}$ : Conjunto de líneas a reconfigurar

\section{Variables:}

$w_{a_{k} e_{k}, 1}$ : Línea entre barra existente y el punto de reconfiguración a

$w_{b_{k} e_{k}, 1}$ : Línea entre barra existente y el punto de reconfiguración b

$w_{a_{k} b_{k}, 1}$ : Línea entre los puntos de reconfiguración a y $b$

El modelo formado por las ecuaciones (1) a (14) corresponde al modelo propuesto en este artículo para el PERT en sistemas eléctricos de potencia considerando reconfiguraciones de red. 


\section{PRUEBAS Y RESULTADOS}

Para verificar la aplicabilidad el modelo propuesto se han considerado dos sistemas de prueba: el sistema Garver de 6 barras y un caso de estudio aplicado al sistema colombiano. A continuación se presentan los resultados para ambos casos.

\section{Pruebas en el sistema Garver de 6 barras}

El Sistema Garver se planteó en (Garver, 1970) y consiste en un sistema de 6 barras y 15 corredores, al cual se le desea encontrar la inversión óptima de mínimo costo para su expansión. En este sistema se permite adicionar hasta 5 líneas por corredor. La mejor solución conocida para este sistema, considerando candidatos convencionales, es de 110 US\$. Tal solución se consigue adicionando una línea entre las barras 3 y 5 , y tres líneas entre las barras 4 y 6 .

Para realizar pruebas del modelo propuesto en el sistema Garver, además de los candidatos tradicionales del problema, se propone incluir como un candidato la reconfiguración de la línea existente 2-4 para la conexión de la barra 6 . En la figura 3 se presentan los parámetros de la reconfiguración propuesta como candidata de expansión, incluyendo dos nuevos nodos ficticios 7 y 8 que representan el punto donde se establece la reconfiguración de la línea 2-4. Con el modelo propuesto y la reconfiguración candidata en el modelo de Garver, se realiza una primera prueba obteniendo la solución que se presenta en tabla 2.

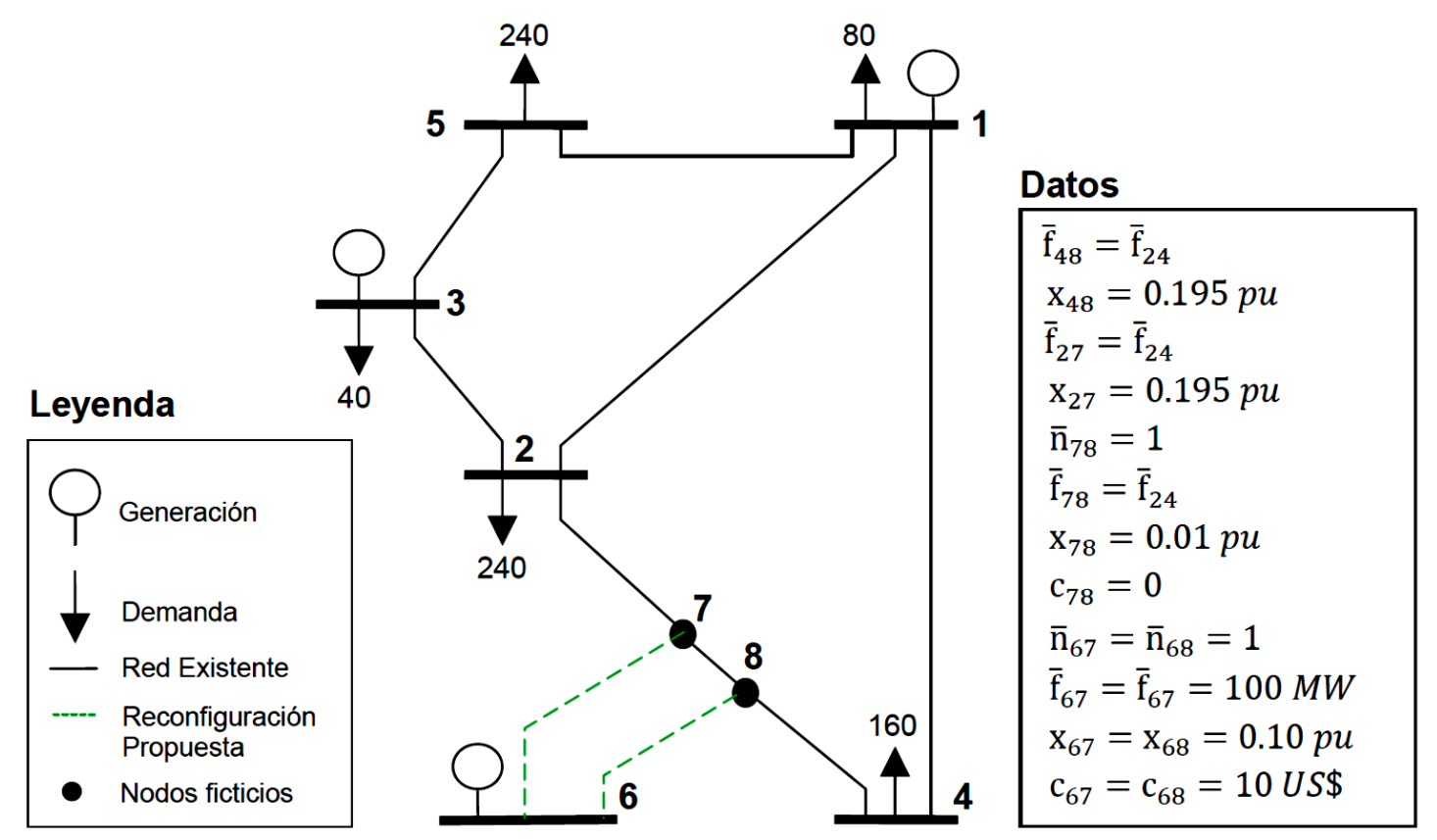

Fig. 3: Parámetros para el candidato de reconfiguración.

Para esta prueba el costo total de la inversión es de 90US. En la tabla 2 se puede observar que en la solución óptima se considera la reconfiguración dentro del plan de expansión del sistema (circuitos nuevos en los corredores 6-7 y 6-8). Adicional a la reconfiguración se observa que en la solución se incluye una segunda línea entre 3-5 y una nueva línea entre 4-6. Lo anterior permite, con los supuestos establecidos, tener un costo total del plan de expansión de 90US\$, 20US\$ menos que la mejor solución conocida del sistema Garver original con candidatos convencionales. Para complementar la prueba anterior y validar el modelo, una segunda prueba es realizada suponiendo que el costo de la reconfiguración es 10 veces al presentado en la figura 3, en cuyo caso el resultado no debe considerar la reconfiguración por su alto costo respecto a los demás candidatos que se tienen disponibles. Los resultados de la segunda prueba se presentan en la tabla 3.

Para esta prueba el costo total de la inversión es de 110US\$, además se puede ver de los resultados de la tabla 3, que cuando el costo de la reconfiguración es muy superior al costo de los candidatos tradicionales, la solución óptima no considera la reconfiguración por su costo y encuentra la mejor solución conocida para el sistema de Garver usando solo candidatos tradicionales. 
Tabla 2. Resultados sistema Garver con reconfiguración - prueba 1.

\begin{tabular}{|c|c|c|c|c|c|}
\hline Nodo i & Nodo $\mathrm{j}$ & Circuitos Existentes & Circuitos Nuevos & Flujo Corredor [MW] & Flujo Máximo [MW] \\
\hline 1 & 2 & 1 & 0 & 13.69 & 100 \\
\hline 1 & 4 & 1 & 0 & 2.61 & 80 \\
\hline 1 & 5 & 1 & 0 & 53.69 & 100 \\
\hline 2 & 3 & 1 & 1 & -133.69 & 200 \\
\hline 3 & 5 & 1 & 1 & 186.31 & 200 \\
\hline 4 & 6 & 0 & 1 & -78.03 & 100 \\
\hline 2 & 7 & 1 & 0 & -92.61 & 100 \\
\hline 8 & 4 & 1 & 0 & 79.36 & 100 \\
\hline 6 & 7 & 0 & 1 & 92.61 & 100 \\
\hline 6 & 8 & 0 & 1 & 79.36 & 100 \\
\hline
\end{tabular}

Tabla 3. Resultados sistema Garver con reconfiguración - prueba 2.

\begin{tabular}{|c|c|c|c|c|c|}
\hline Nodo i & Nodo $\mathrm{j}$ & Circuitos Existentes & Circuitos Nuevos & Flujo Corredor [MW] & Flujo Máximo [MW] \\
\hline 1 & 2 & 1 & 0 & 40.00 & 100 \\
\hline 1 & 4 & 1 & 0 & -40.00 & 80 \\
\hline 1 & 5 & 1 & 0 & 66.67 & 100 \\
\hline 2 & 3 & 1 & 0 & -100.00 & 200 \\
\hline 3 & 5 & 1 & 1 & 173.33 & 200 \\
\hline 4 & 6 & 0 & 3 & -300.00 & 100 \\
\hline 2 & 7 & 1 & 0 & -100.00 & 100 \\
\hline 7 & 8 & 0 & 1 & -100.00 & 100 \\
\hline 8 & 4 & 1 & 0 & -100.00 & 100 \\
\hline
\end{tabular}

\section{Aplicación en un sistema real colombiano}

El generador de TERMOCOL se conectará en Colombia en el área de Guajira-Cesar-Magdalena (GCM), con una capacidad de 202MW cerca de la ciudad de Santa Marta en la costa caribe. Para la conexión de este generador al sistema Colombiano en el documento (UPME, 2012) se evaluaron diferentes alternativas de conexión. En las figuras 4 y 5 se ilustra la red y las alternativas analizadas, respectivamente.

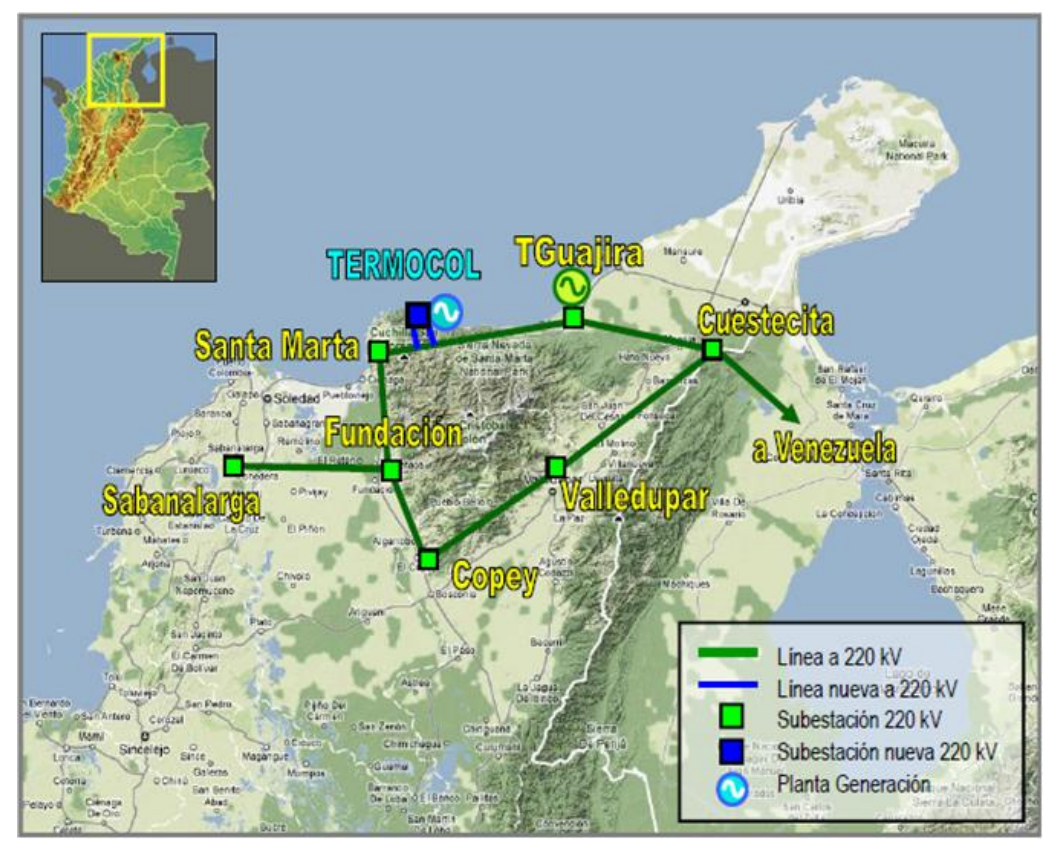

Fig. 4: Diagrama del área GCM para la conexión de TERMOCOL, Fuente UPME. 


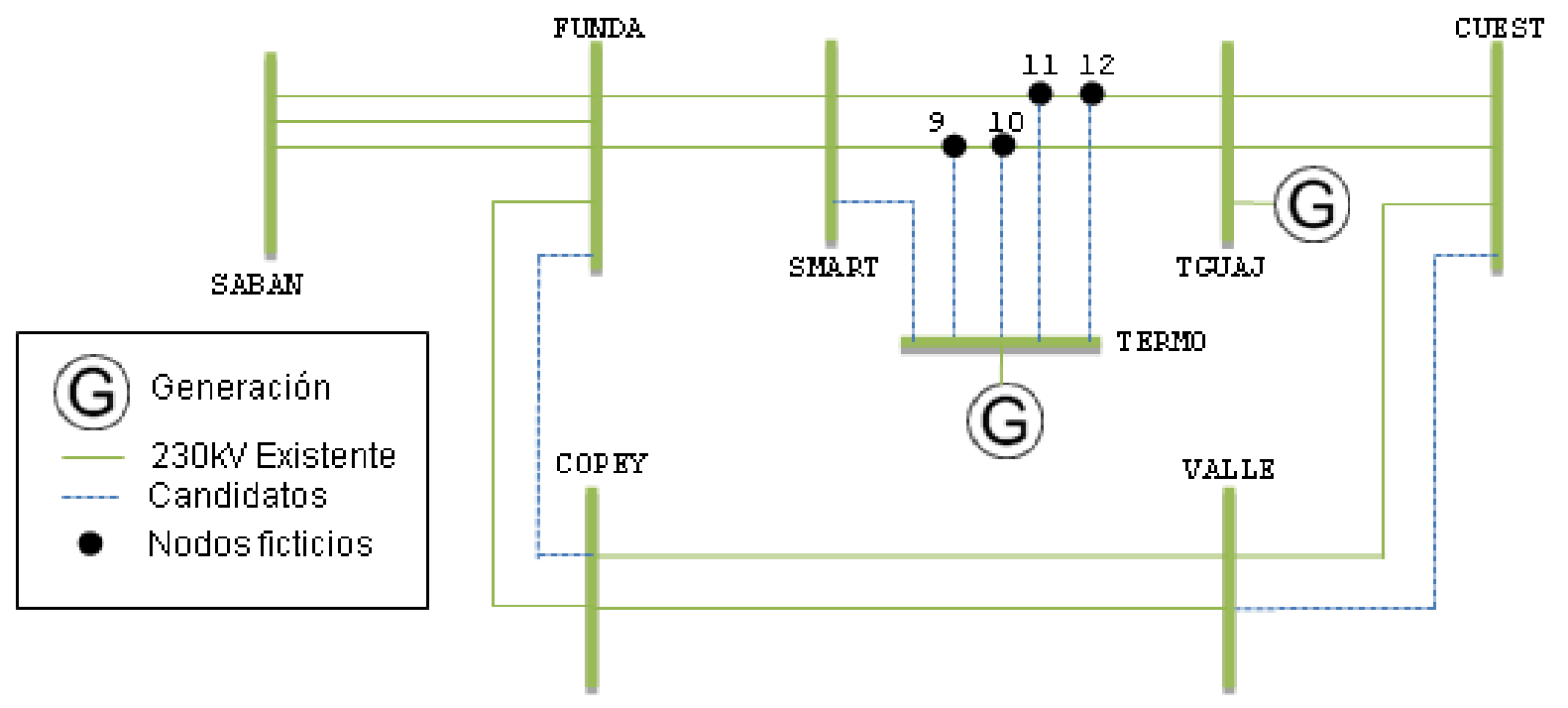

Fig 5. Alternativas para la conexión de TERMOCOL.

Para la implementación de este problema las barras del número 9 al 12 son las barras creadas para la consideración de las reconfiguraciones planteadas para los circuitos 1 y 2 Santa Marta - Termoguajira $230 \mathrm{kV}$. En el área de GCM las barras en las cuales se encuentra la demanda son Cuestecitas (CUEST), Valledupar (VALLE), Copey (COPEY), Fundación (FUNDA) y Santa Marta (SMART). Las barras de Sabanalarga (SABAN) y Copey son nodos de conexión con otras áreas y pueden inyectar potencia al área, cuando la generación interna es menor a la demanda, o puede llevar la potencia del área GCM a otras áreas del país, cuando la generación interna es mayor a la demanda interna. La barra de Cuestecitas es un nodo que sirve como conexión internacional con Venezuela y en esta barra es posible tanto importar como exportar energía desde el país vecino. Para las pruebas al modelo se analizó un escenario de demanda máxima en el área con solo el generador de TERMOCOL en servicio. En este escenario las barras de Copey y Sabanalarga se comportan como generadores debido a que se encuentran inyectando potencia al área de GCM. En la tabla 4, se presentan los resultados obtenidos.

Tabla 4. Resultados expansión para TERMOCOL

\begin{tabular}{|c|c|c|c|c|c|}
\hline Nodo $\mathrm{i}$ & Nodo $\mathrm{j}$ & Circuitos Existentes & Circuitos Nuevos & Flujo Corredor [MW] & Flujo Máximo [MW] \\
\hline CUEST & TGUAJ & 2 & 0 & -101.60 & 452 \\
\hline CUEST & VALLE & 1 & 0 & 1.60 & 214 \\
\hline VALLE & COPEY & 2 & 0 & -138.40 & 422 \\
\hline COPEY & FUNDA & 1 & 0 & -85.40 & 214 \\
\hline FUNDA & SMART & 2 & 0 & 49.60 & 470 \\
\hline SABAN & FUNDA & 3 & 0 & 180.00 & 912 \\
\hline SMART & 9 & 1 & 0 & -145.06 & 235 \\
\hline 10 & TGUAJ & 1 & 0 & 56.94 & 235 \\
\hline SMART & 11 & 1 & 0 & 44.67 & 235 \\
\hline 11 & 12 & 0 & 1 & 44.67 & 235 \\
\hline 12 & TGUAJ & 1 & 0 & 44.67 & 235 \\
\hline TERMO & 9 & 0 & 1 & 145.06 & 344 \\
\hline TERMO & 10 & 0 & 1 & 56.94 & 344 \\
\hline
\end{tabular}

La solución óptima encontrada bajo este escenario es la reconfiguración de la línea Termoguajira - Santa Marta 230kV en las líneas Termoguajira - Termocol 230kV y Termocol - Santa Marta 230kV respectivamente. El costo de esta solución es de 0.02MUSD, el cual es debido a la longitud de las líneas asociadas a la reconfiguración. En la figura 6 , se presenta en forma gráfica la solución encontrada. 


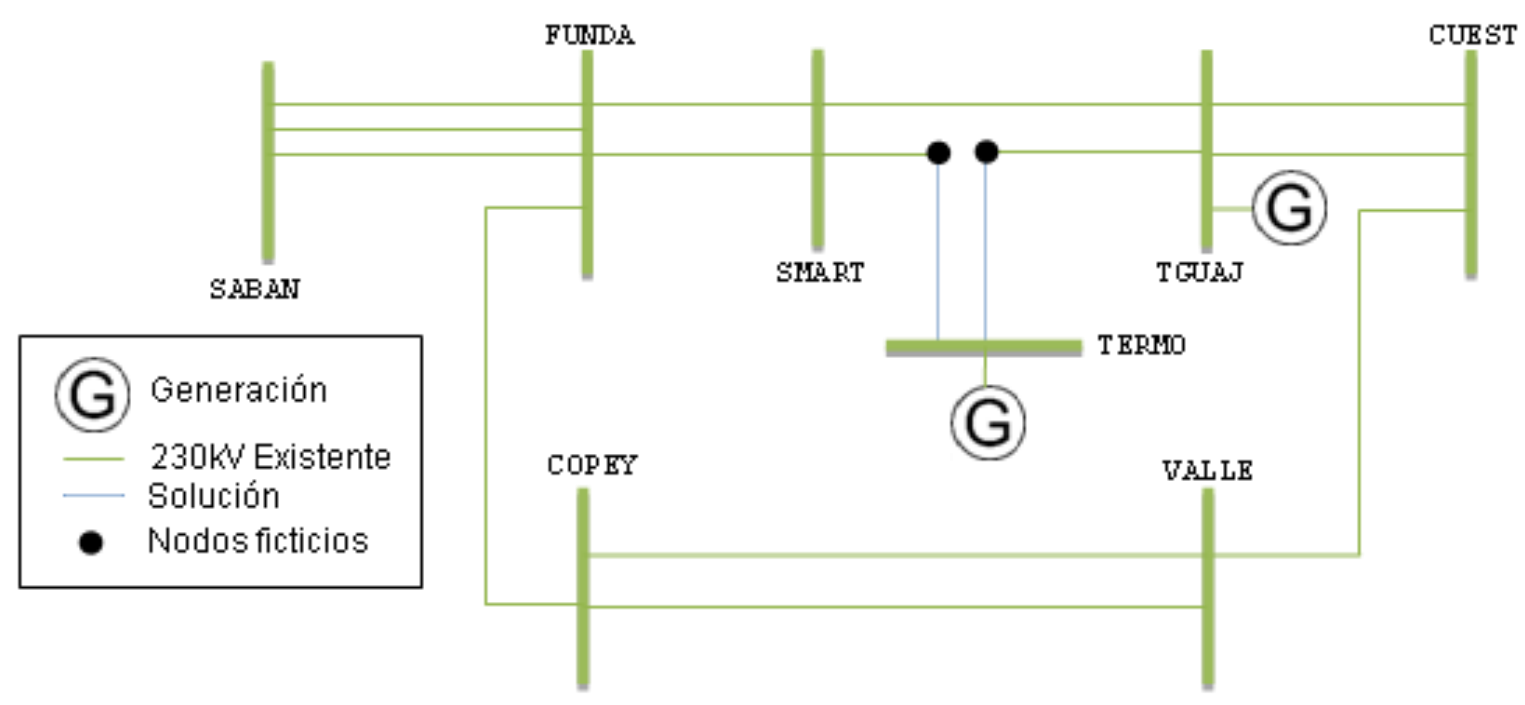

Fig 6. Resultado conexión del generador TERMOCOL.

De no haberse considerado como alternativa de solución la reconfiguración de las líneas existentes, la solución óptima sería la construcción de un circuito entre las subestaciones Termocol (TERMO) y Santa Marta (SMART) con un costo total de 1.05MUSD. Se observa entonces como la solución mediante la consideración de reconfiguraciones reduce la inversión total en cerca de 1.03MUSD, cumpliendo el mismo objetivo de permitir la conexión del generador al sistema.

\section{CONCLUSIONES}

En este artículo se han presentado las ventajas de tener en un modelo de optimización la posibilidad de contar con reconfiguraciones de red. Incluir este tipo de candidatos ha permitido explorar nuevos espacios de búsqueda para el PERT, sobre los cuales se han encontrado mejores soluciones a las obtenidas utilizando únicamente candidatos convencionales. La reconfiguración de redes existentes ha mostrado ser una solución factible en los sistemas de potencia alrededor del mundo. Por tal razón, contar con un modelo de optimización para el PERT que incluya candidatos convencionales y reconfiguraciones permite dar mejores señales de expansión en las redes de transmisión de los sistemas eléctricos de potencia.

El haber desarrollado el modelo mediante restricciones lineales y variables binarias usando el modelo lineal disyuntivo, permite mantener las condiciones para que la solución del PERT, en cada uno de los casos, sea el óptimo global del problema.

Con el modelo planteado en este artículo y otros desarrollos que se encuentran en la literatura, es posible crear, en un trabajo futuro, un nuevo modelo más completo que considere adicional a los candidatos no convencionales, contingencias en la red, múltiples escenarios de generación y demanda y múltiples etapas de planeamiento.

\section{AGRADECIMIENTOS}

Los autores agradecen a la Universidad de Antioquia (UdeA) por el apoyo del proyecto "Sostenibilidad 20132014".

\section{REFERENCIAS}

ALTALINK, Windy Flats 138kV Line Re-configuration, http://www.altalink.ca/projects/ southernabtransmission/sabtransmissionreinforcement/satr-windy-flats.cfm. Acceso: 3 de abril (2013)

Anaut D. O., Di Mauro G. F., Meschino G. y Suárez J. A., Optimización de Redes Eléctricas Mediante la Aplicación de Algoritmos Genéticos, Revista Información Tecnológica: 20(4), 137-148 (2009)

Areiza, J. M., Luiz da Silva, E., Couto de Oliveira, G., y Binato, S., Transmission Network Expansion Planning Under a Tabu Search Approach, IEEE Transactions on Power Systems: 16(1), 62-68 (2001) 
Bahiense, L., Oliveira, G. C., Pereira, M., y Granville, S., A mixed integer disjunctive model for transmission network expansion, IEEE Transactions on Power Systems: 16(3), 560-565 (2001)

Chen, Y., Chen, H., Chen, J., y Duan, X., An Improved Particle Swarm Optimization Algorithm for Multistage and Coordinated Planning of Transmission Systems, Transmission and Distribution Conference and Exhibition IEEE/PES, Asia and Pacific, (2005)

Escobar, A. H., Gallego, R. A., y Romero, R., Multistage and coordinated planning of the expansion of transmission systems, IEEE Transactions on Power Systems: 16(3), 560-565 (2001)

Gallego, L., Rider, M. J., Romero, R., y García, A., A Specialized Genetic Algorithm to Solve the Short Term Transmission Network Expansion Planning, IEEE Bucharest Power Tech Conference, Bucharest, (2009)

Gallego, R. A., Monticelli, A., y Romero, R., Transmision system expansion planning by an extended genetic algorithm, IEE Proc.-Gener. Transm. Distrib: 145(3), 329-335 (1998)

Gallego, R., Romero, R., y Monticelli, A., Tabu search algorithm for network synthesis, IEEE Transactions on Power Systems: 15(2), 490-495 (2000)

García, C., García, E., y Villada, F., Algoritmo Evolutivo Eficiente Aplicado a la Planeación de la Expansión de Sistemas de Distribución, Información tecnológica: 23(4), 3-10 (2012)

Garver, L. L., Transmission network estimation using linear programming, IEEE Transactions on Power Apparatus and Systems: PAS-89(7), 1688-1697 (1970)

Kazerooni, A., y Mulate, J., Transmission Network Planning Under Security and Environmental Constraints, IEEE Transactions on Power Systems: 25(2), 1169-1178 (2010)

Latorre, G., Cruz, R., Areiza, J., y Villegas, A., Classification of publications and models on transmission expansion planning, IEEE Transactions on Power Systems: 18(1), 938-946 (2003)

Luiz da Silva, E., Gil, H. A., y Areiza, J. M., Transmission Network Expansion Planning Under an Improved Genetic Algorithm, IEEE Transactions on Power Systems: 15(3), 1168-1175 (2000)

Molina, J. D., y Rudnick, H., Transmission of Electric Energy: A Bibliographic Review, IEEE Latin America Transactions: 8(3), 245-258 (2010)

Oliveira, G., Binato, S., y Pereira, M., Value-Based Transmission Expansion Planning of Hydrothermal Systems Under Uncertainty, IEEE Transactions on Power Systems: 22(4), 1429-1435 (2007)

Peng, W., Haozhong, C., y Jie, X., The Interval Minimum Load Cutting Problem in the Process of Transmission Network Expansion Planning Considering Uncertainty in Demand, IEEE Transactions on Power Systems: 23(3), 1497-1506 (2008)

Pozo, D., Sauma, E. E., y Contreras, J., A Three-Level Static MILP Model for Generation and Transmission Expansion Planning, IEEE Transactions on Power Systems: 28(1), 202-210 (2013)

Rider, M. J., García, A. V., y Romero, R., Power system transmission network expansion planning using AC model, The Institution of Engineering and Technology: 1(5), 731-742 (2007)

Rider, M., Garcia, A., y Romero, R., Transmission system expansion planning by a branch-and-bound algorithm, IET Generation, Transmission \& Distribution: 2(1), 90-99 (2008)

Romero, N., Xu, N., Nozick, L., Dobson, I., y Jones, D., Investment Planning for Electric Power Systems Under Terrorist Threat, IEEE Transactions on Power Systems: 27(1), 108-116 (2012)

Romero, R., Monticelli, A., García, A., y Haffner, S., Test systems and mathematical models for transmission network expansion planning, IEE Proc. Gener. Transm. Distrib: 149(1) (2002) 
Romero, R., Rocha, C., Mantovani, M., y Mantovani, J., Analysis of heuristic algorithms for the transportation model in static and multistage planning in network expansion systems, Generation, Transmission and Distribution IEE Proceedings: 150(5), 521-526 (2003)

Sharifnia, A., y Aashtiani, H., Transmission Network Planning: A Method for Synthesis of Minimum-Cost Secure Networks, IEEE Transactions on Power Apparatus and Systems: PAS-104(8), 2025-2034 (1985)

Silvia, I. J., Rider, M. J., y Romero, R., Transmission network expansion planning with security constraints, IEE Proc. GEner. Transm. Distrib: 152(6) (2005)

Silvia, I. J., Rider, M. J., y Romero, R., Transmission network expansion planning considering uncertainness in demand, IEEE Transactions on Power Systems: 21(4), 1565-1573 (2006)

UPME, Plan de Expansión de Referencia Generación - Transmisión 2012-2025, http://www.upme.gov.co/Docs/Plan_Expansion/2012/PLAN_COMPLETO_2012-2025.pdf. Acceso: 20 de octubre (2012)

Villasana, R., Garver, L. L., y Salon, S. J., Transmission network planning using linear programming, IEEE Transactions on Power Apparatus and Systems: PAS-104(2), 349-356 (1985)

Vinasco, G., Rider, M. J., y Romero, R., A strategy to solve the multistage transmission expansion planning problem, IEEE Transactions on Power Systems: 26(4), 2574-2576 (2011)

Zhang, H., Vittal, V., Heydt, G., y Quintero, J., A Mixed-Integer Linear Programming Approach for MultiStage Security-Constrained Transmission Expansion Planning, IEEE Transactions on Power Systems: 27(2), 1125-1133 (2012) 
